\title{
Determination of Precision Fusion Cross Sections Using a High Efficiency Superconducting Solenoidal Separator
}

\author{
L. T. Bezzina ${ }^{1} \star \star$, E. C. Simpson ${ }^{1}$, I. P. Carter ${ }^{1}, \quad$ M. Dasgupta ${ }^{1}$, T. Ebadi ${ }^{1}$, D. J. Hinde ${ }^{1}$, and D. C. Rafferty ${ }^{1}$ \\ ${ }^{1}$ Department of Nuclear Physics, Research School of Physics and Engineering, The Australian National University, Acton ACT \\ 2601, Australia
}

\begin{abstract}
A novel fusion product separator based on a gas-filled superconducting solenoid has been developed at the Australian National University. Though the transmission efficiency of the solenoid is very high, precision cross sections measurements require this efficiency to be estimated accurately. A Monte Carlo simulation for the efficiency can be performed, but in turn requires knowledge of the angular distribution of the evaporation residues. We have developed a method to deduce the angular distribution of the evaporation residues from the laboratory-frame velocity distribution $\sigma_{\text {exp }}\left(v_{l}\right)$ of the evaporation residues measured after the solenoid. The method will be discussed, focussing on the example of ${ }^{34} \mathrm{~S}+{ }^{89} \mathrm{Y}$, where the angular distribution has been independently measured using a velocity filter [1]. The establishment of this method now allows the novel solenoidal separator to be used to obtain the most reliable, precision fusion cross-sections.
\end{abstract}

\section{Introduction}

The study of heavy-ion fusion gives us insights into nuclear structure and many-body quantum dynamics. With applications from the production of medical isotopes to the creation of superheavy elements, accurate knowledge of the cross sections of such fusion reactions is vital in order to optimise the product yield.

For light nuclei, good agreement between experiment and theory is found utilising simple barrier-passing models of fusion. However, studies of the fusion of heavier nuclei revealed an enhanced cross section at below-barrier energies [2-5]. The explanation of this enhancement was provided by the quantum coupled-channels model of fusion [6-9], which introduced the concept of multiple barriers resulting fromthe quantum superposition of target and projectile states. Measurements presented in 2007 [10], however, revealed the shortcomings of current coupledchannels models, notably the failure to provide simultanaeous prediction of above- and below-barrier cross sections.

This inconsistency of the coupled-channels model suggests a fundamental process is being overlooked theoretically. In order to accommodate this inconsistency, a number of explanations have been put forward, including effects of energy dissipation both below and above the barrier [10], and the damping of quantum vibrations as the single-particle wavefunctions of the two nuclei mix [11]. The role of the incompressibility of nuclear matter has also been explored [12], while more recently, a microscopic approach to include the effects of Pauli repulsion has also been developed [13]. As modifications to the existing the-

\footnotetext{
^e-mail: lauren.bezzina@anu.edu.au
}

ory are suggested and new models of fusion arise, verification of these theories via experimental measurements will be required.

The most strenuous experimental tests for new theoretical models will be provided by measurements of the fusion barrier distribution, requiring extremely precise cross section measurements. The novel fusion product separator based on a gas-filled superconducting $8 \mathrm{~T}$ solenoid, developed at the Australian National University (ANU), is well-placed to make these measurements.

This paper describes the development of a method to extract the angular distribution of fusion products, correcting for the transmission efficiency of the solenoid. Section 2 introduces the solenoidal separator, giving context to the iterative correction procedure described in section 3 . Comparison of the deduced angular distribution with dedicated measurements and statistical model calculations is covered in section 4. Final conclusions and future directions of study conclude this paper in section 5 .

\section{Superconducting Solenoidal Separator}

The $8 \mathrm{~T}$ superconducting SOLenoid for In-beam Transport And Identification of Recoiling Evaporation-residues, SOLITAIRE, is a new high efficiency fusion product separator at the ANU, used to ensure spatial separation of the high flux of elastically scattered beam particles from the evaporation residue (ER) fusion products. A schematic of the SOLITAIRE system is presented in figure 1 .

Following the interaction of the beam with the target foil, the elastically scattered beam and ERs must first pass through the soft iron nose cone making up part of the iron shielding that encases the solenoid. The nose cone limits 


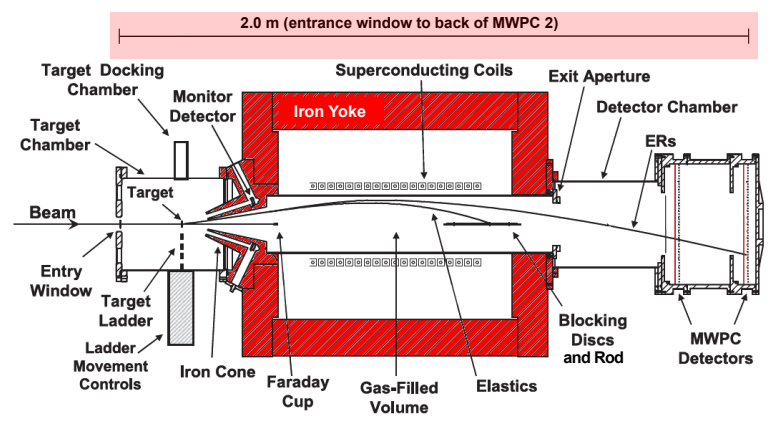

Figure 1: Schematic cross section of SOLITAIRE [14] showing radial trajectories of elastically scattered beam and ERs.

the angular acceptance of SOLITAIRE to $9.5^{\circ}$. Beyond this nose cone, the unscattered beam that passes through the target is collected by a Faraday Cup at the solenoid entrance which subtends an angle of less than $1^{\circ}$. The ERs are deflected away from the beam axis by the recoil effect of the evaporation process, ensuring the loss of ERs to the Faraday Cup is minimal.

Those elastically scattered beam particles and ERs not collected by the Faraday Cup continue to travel through the gas-filled volume of the solenoid. Prior to entering the solenoid, these particles have a distribution of charge states due to interactions with the thin target foil. In the solenoid, interactions with the dilute helium gas mean that an equilibrium mean charge state is quickly reached. It is the difference in average velocity (lower for the ERs compared to the elastically scattered beam), which allows the elastically scattered particles to be brought to a focus before the ERs, allowing them to be physically blocked using a blocking rod and series of blocking discs on the solenoid axis.

The ERs are brought to a focus further downstream, beyond the exit of the solenoid bore. Here they are detected using SOLITAIRE's two large $(20$ x $20 \mathrm{~cm})$ position sensitive multiwire proportional gas counters (MWPCs). These detectors allow the identification of the detected particles from their energy loss and time of flight information, from which the velocity distribtion of the ERs can be determined.

\section{Iterative Procedure to Deduce Angular Distribution}

The extraction of ER cross sections requires a precise determination of the solenoid's efficiency. In turn, this requires an understanding of the angular distribution of the evaporation residues exiting the target. A method has been developed to deduce the angular distribution from the measured laboratory-frame velocity distribution [15] of the ERs exiting the solenoid.

This method is based on the relationship between the velocity vectors of the evaporation residue following emission in the laboratory frame, and centre-of-mass frame, depicted in figure 2, and denoted with a subcript $l$ and $c$, re-

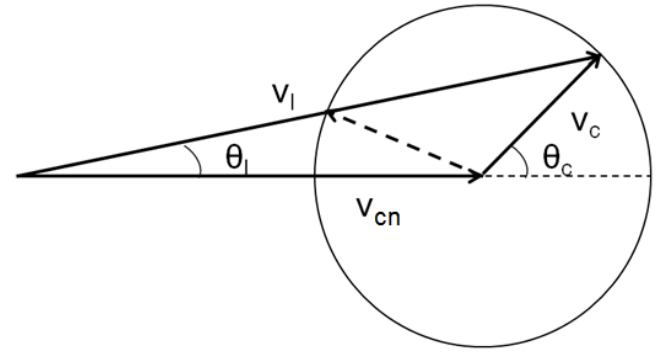

Figure 2: Velocity vector diagram illustrating the relationship between evaporation residue laboratory velocity $\left(v_{l}\right)$ and angle $\left(\theta_{l}\right)$ and centre of mass velocity $\left(v_{c}\right)$ and angle $\left(\theta_{c}\right)$, through the laboratory-frame compound nucleus velocity $v_{c n}$.

spectively. These are related through the laboratory frame compound nucleus velocity, $v_{c n}$. It is clear from this diagram that both the laboratory-frame velocity distribution, $\sigma\left(v_{l}\right)$ and angular distribution, $\sigma\left(\theta_{l}\right)$ are related by the velocity distribution in the centre-of-mass frame, $\sigma\left(v_{c}\right)$. By assuming an isotropic form of the centre-of-mass angular distribution $\sigma\left(\theta_{c}\right)$, extraction of $\sigma\left(v_{c}\right)$ from $\sigma\left(v_{l}\right)$ can be performed. Once $\sigma\left(v_{c}\right)$ is known, it can be used to construct a variety of cross section expressions, including the $2 \mathrm{D}$ cross section as a function of laboratory angle and velocity, $\sigma\left(v_{l}, \theta_{l}\right)[15]$.

The measured $\sigma_{\text {exp }}\left(v_{l}\right)$, however, is moderated by the transmission efficiency of the solenoid. The efficiency will clearly depend on $\theta_{l}$ due to the physical obstruction of the Faraday Cup and the nose cone. Since changes in $v_{l}$ change the corresponing magnetic rigidity, there is also a potential $v_{l}$ dependence. It is thus necessary to correct for the efficiency of the solenoid as a function of both $v_{l}$ and $\theta_{l}$.

Testing of this method to include the effects of transmission efficiency was achieved using existing measurments of the velocity distribution of the reaction of a $124 \mathrm{MeV}{ }^{34} \mathrm{~S}$ beam on a ${ }^{89} \mathrm{Y}$ target of thickness $100 \mu \mathrm{g} / \mathrm{cm}^{2}$. This reaction was chosen because it not only has a well-known fusion cross section measured previously, but there are also existing, reliable direct measurements of the angular distribution. These measurements, made by Mukherjee et al. [1], were made possible by scanning in angle using a velocity filter, as detailed by Wei et al. [16]. While more direct, measuring the angular distribution in this way is far more time-consuming, taking a number of days to collect enough counts for satistfactory statistics at all angles.

In order to take the transmission efficiency of the solenoid into account, simulations of the transport of the ERs through the solenoid were performed using the simulation software Solix. Solix is a Monte Carlo based $\mathrm{C}++$ code developed at the Australian National University [17], designed to replicate as closely as possible the SOLITARE setup. These simulations take as input the laboratory-frame angle and velocity of the ERs, and can be used to generate a two-dimensional distribution of the transmission efficiency as a function of these two parame- 


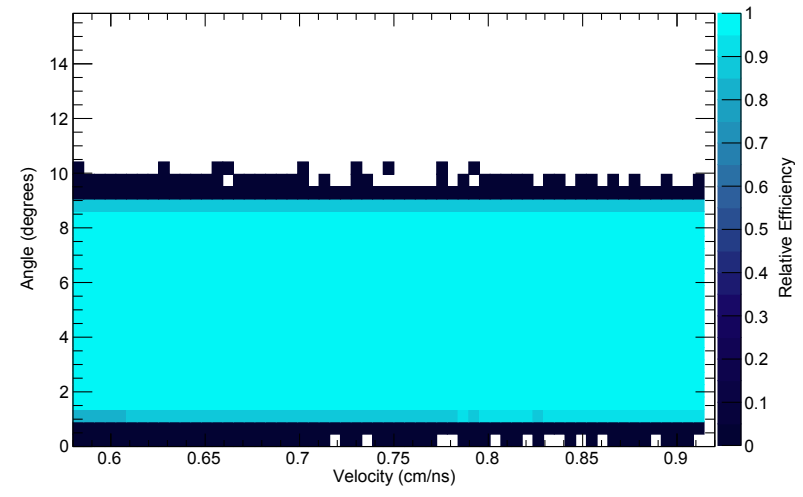

Figure 3: Transmission efficiency distribution as a function of $v_{l}$ and $\theta_{l}$ used for the corrective iteration procedure.

ters, $\varepsilon\left(v_{l}, \theta_{l}\right)$. The efficiency distribution for the $124 \mathrm{MeV}$ ${ }^{34} \mathrm{~S}+{ }^{89} \mathrm{Y}$ reaction is shown in figure 3. The uniformity in $v_{l}$ is a direct result of the presence of the gas filling the solenoid; since the mean charge state is assumed to depend linearly on $v_{l}$ [18], the magnetic rigidity is independent of $v_{l}$, and thus the efficiency is also. This distribution can then be used in an iterative correction procedure to moderate the $2 \mathrm{D}$ cross section $\sigma\left(v_{l}, \theta_{l}\right)$.

The basis of this procedure is a parameterised form of the centre-of-mass velocity distribution, $\sigma\left(v_{c}\right)$. In the first instance, this distribution is formed from the laboratoryframe velocity distribution $\sigma_{\text {exp }}\left(v_{l}\right)$ using the analytical transformations of Reference [15].

The $\sigma\left(v_{c}\right)$ distribution is then parameterised using the sum of a number of Maxwell-Boltzmann functions. This distribution was chosen following the arguments established by Weisskopf [19] to model the deexcitation of the compound nucleus using the statistical model. Initial calculation of $\sigma\left(v_{c}\right)$ from the experimental $\sigma\left(v_{l}\right)$ (seen in figure 4(a)) suggests that, despite statistical fluctuations giving some irregularity, the distribution takes the shape of the sum of two Maxwell-Boltzmann-like distrbutions, one a true Maxwell-Boltzmann distribution, the other with a similar shape, but a non-zero offset of the distribution. This form suggests two dominant emission modes, one with no threshold to emission, and the other with a potential threshold to emission, resulting in the nonzero offset. These distributions can be related to neutron and charged particle evaporation respectively [20].

The parameterised $\sigma\left(v_{c}\right)$ is then used to generate the two-dimensional distribution $\sigma\left(v_{l}, \theta_{l}\right)$. This distribution is filtered by the transmission efficiency $\varepsilon\left(v_{l}, \theta_{l}\right)$ by mutiplying the two distributions in a bin-by-bin manner. Following this moderation by efficiency, $\sigma\left(v_{l}, \theta_{l}\right)$ is then projected onto the $v_{l}$ axis to produce the distribtion $\sigma\left(v_{l}\right)$. This derived distribution can then be compared to the experimental velocity distribution $\sigma_{\text {exp }}\left(v_{l}\right)$ numerically using the $\chi^{2}$ parameter.

This enables the design of an iterative procedure, based on the minimisation of the $\chi^{2}$ parameter by varying the input parameters of the original centre-of-mass veloc-
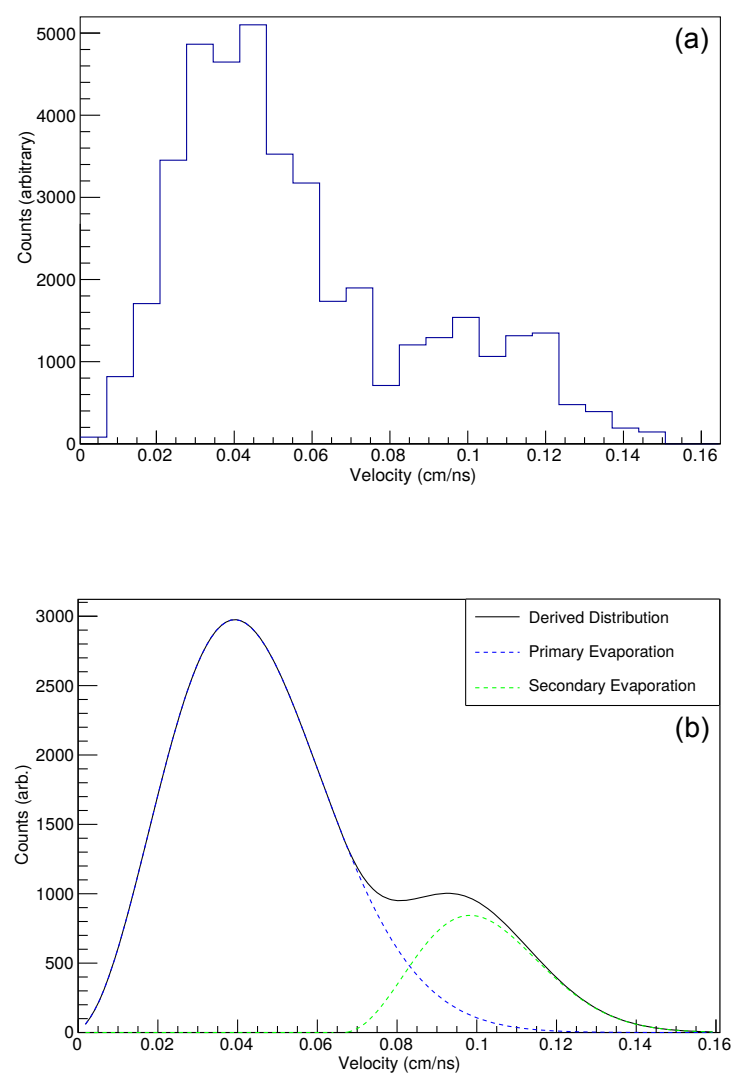

Figure 4: Comparison of initial extracted and final parameterised $\sigma\left(v_{c}\right)$ distribution. (a) $\sigma\left(v_{c}\right)$ distribution obtained by boot-strap extraction from the measured $\sigma_{\text {exp }}\left(v_{l}\right)$ data. (b) Final parameterised $\sigma\left(v_{c}\right)$ produced by minimising the $\chi^{2}$ between $\sigma_{\text {exp }}\left(v_{l}\right)$ and calculated $\sigma\left(v_{l}\right)$. Note that the yaxis scales are arbitrary, and differ due to the moderation of (a) by the transmission efficiency of the solenoid.

ity distribution $\sigma\left(v_{c}\right)$. Once the minimum $\chi^{2}$ has been reached, the $v_{c}$ distribution produced can be taken as the true $\sigma\left(v_{c}\right)$ distribution, as it correctly reproduces the measured $\sigma_{\text {exp }}\left(v_{l}\right)$ distribution after being passed through the efficiency of the solenoid. Figure 5 shows the deduced $\sigma\left(v_{l}\right)$ that gave the minimum $\chi^{2}$ value alongside the experimental $\sigma_{\text {exp }}\left(v_{l}\right)$, while figure $4(\mathrm{~b})$ shows the parameterised $\sigma\left(v_{c}\right)$ which gave this best fit.

\section{Deduced Angular Distribution}

Following the derivation of this true $\sigma\left(v_{c}\right)$, a final calculation can be performed to obtain the two-dimensional cross section $\sigma\left(v_{l}, \theta_{l}\right)$ which can then be projected onto the $\theta_{l}$ axis to obtain the laboratory-frame angular distribution $\sigma\left(\theta_{l}\right)$. This distribution can then be normalised in order to present the absolute fusion cross sections of the reaction in question. This normalisation is made possible through SOLITAIRE's silicon monitor detectors, placed at fixed, known angles before the entrance of the solenoid in order to detect the elastically scattered beam particles. Nor- 


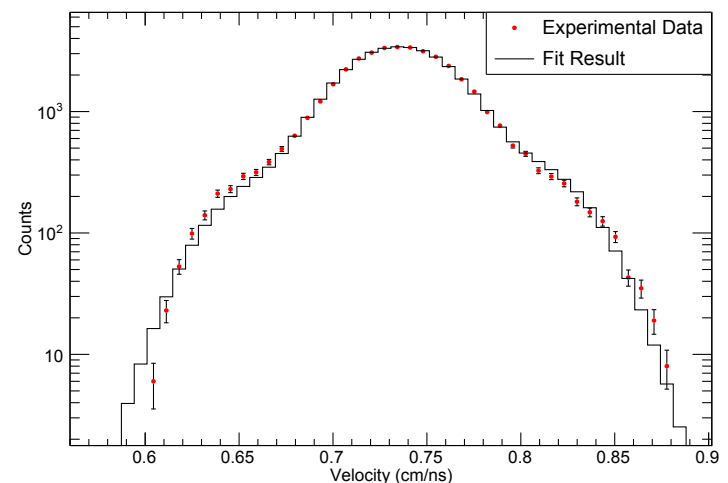

Figure 5: $\sigma\left(v_{l}\right)$ distribution deduced by minimisation routine, shown along with the measured $\sigma_{\text {exp }}\left(v_{l}\right)$ data set.

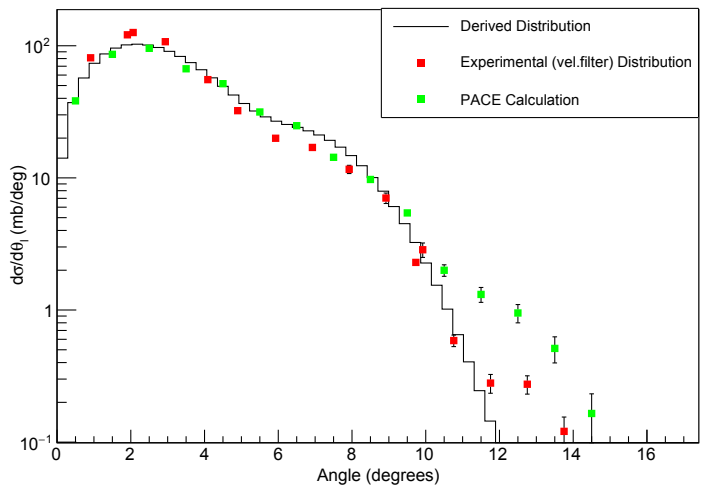

Figure 6: Final $\sigma\left(\theta_{l}\right)$ produced by the corrective minimisation routine, shown alongside direct angular distribution measurements (red squares) [1] and PACE4 statistical model calculations (green squares). Despite being too small to see, the measurements from reference [1] include experimental error bars, while the PACE4 data points include error bars defined by the square root of the counts, as is standard for Monte Carlo simulations.

malisation of the distribution allows the derived angular distribution to be compared with the dedicated experimental measurements made by Mukherjee et al. [1]. An additional comparison with the statistical model deexcitation code PACE4 [21] (included in the program LISE++ [22]) is included, as seen in figure 6 .

It is immediately clear that there is good agreement of the primary peak position (at low angles) between the data and two sets of calculations. The magnitude of the primary peak, however, is underestimated in both the derived distribution and in the PACE4 calculations when compared to the experimental data. There is also a significant overestimation of the experimental cross section between $5^{\circ}$ and $10^{\circ}$. This suggests an issue with the parameterised $\sigma\left(v_{c}\right)$, the efficiency calculation, or with the measured velocity distribution $\sigma_{\text {exp }}\left(v_{l}\right)$.
Underestimation of the peak at small angles suggests that counts from the high- and low-velocity edges of the distribution are missing, which could be put down to the initial graphical cuts made to the energy vs. time of flight data set made to select out the ERs for the creation of the $\sigma_{\text {exp }}\left(v_{l}\right)$ distribution. Considering also the overestimation of the cross section at the mid-range angles, however, suggests that the counts missing from the edges of the velocity distribution may actually be present at velocities near $v_{c n}$, and a systematic issue exists.

This systematic issue may be related to assumptions made about how much energy is lost by the ERs as they traverse the solenoid. The current assumption is that there is negligible energy loss, resulting in $\sigma_{\text {exp }}\left(v_{l}\right)$ being centred precisely on $v_{c n}$. Considering energy losses through the gas will result in a centroid smaller than $v_{c n}$, as well as compressing the distribution at higher velocities (higher energy ERs are subject to greater energy loss than lower energy ERs [23]). This suggests that an investigation into quantifying and correcting for the energy losses through the solenoid is a worthwhile direction for future studies.

The position of the shoulder at $7-8^{\circ}$ is also in good agreement across all three distributions. The magnitude of this second feature, however, again differs across the distributions. There is better agreement between the PACE4 simulation and the distribution produced in this study than with the experimental data. One explanation for this could be the assumption of isotropic evaporation of the particles. While the calculated distribution assumes isotropic evaporation, there may be some anisotropy present in the real evaporation process. For anisotropy favouring the forward and backward emission of particles in the centre-of-mass frame, the number of ERs detected at lower angles will be enhanced compared to isotropic emission. If this assumption was incorporated into the calculated distribution, the peak at low angles would be enhanced compared to that at around $8^{\circ}$, and it would be expected that the agreement between the calculated and experimental data sets would be improved.

The final feature of note is the identification of a third Maxwell-Boltzmann component of the $\theta_{l}$ distribution in the PACE4 calculations. This is prominent in the region of $11-16^{\circ}$, and it indicates the contribution of a third evaporation mode to the overall distribution. Analysis of the PACE4 output data suggests that this third component arises from the emission of two alpha particles [24]. An argument could also be made for this presence of this third component in the experimental distribution, too, but it is not entirely clear whether this shape is a genuine feature or just a statistical fluctuation in the data. During this study, a third Maxwell-Boltzmann was added to the parameterisation in an attempt to test whether this component was indeed contributing in a significant way. It was found, however, that since this component was contributing to the distribution at larger angles, where the efficiency of the solenoid was found to be low, this third MaxwellBoltzmann was not well-constrained enough to give a reliable contribution to the overall distribution. 


\section{Conclusions and Future Work}

The large ER transmission efficiency of the Australian National University's fusion product separator SOLITAIRE has been characterised as a function of laboratory angle and velocity using Monte-Carlo simulations. This efficiency distribution was used alongside a parameterised centre-of-mass frame velocity distribution in the form of a double Maxwell-Boltzmann and the appropriate mathematical transformations to extract the angular distribution of evaporation residues entering the solenoid. The generated angular distribution compares quite well in magnitude and has excellent agreement in overall shape to both dedicated measurements and PACE4 calculations.

In order to improve the agreement between the derived and directly-measured angular distributions, this method will be refined by taking into account energy loss of the ERs with the fill gas as they traverse the solenoid. Additionally, the effect of anisotropy of particle emission in the centre-of-mass frame will be investigated using a parameterised anisotropy dsitribution, where the parameters will be set and varied following physical arguments, and changes to the derived angular distribution will be examined.

\section{References}

[1] A. Mukherjee, M. Dasgupta, D.J. Hinde, K. Hagino, J.R. Leigh, J.C. Mein, C.R. Morton, J.O. Newton, H. Timmers, Phys. Rev. C. 66, 034607 (2002)

[2] R.G. Stokstad, Y. Eisen, S. Kaplanis, D. Pelte, U. Smilansky, I. Tserruya, Phys. Rev. C 21, 2472 (1980)

[3] J.R. Leigh, M. Dasgupta, D.J. Hinde, J.C. Mein, C.R. Morton, R.C. Lemmon, J.P. Lestone, J.O. Newton, H. Timmers, J.X. Wei et al., Phys. Rev. C 52, 3151 (1995)

[4] M. Beckerman, J. Ball, H. Enge, M. Salomaa, A. Sperduto, S. Gazes, A. DiRienzo, J.D. Molitoris, Phys. Rev. C 23, 1581 (1981)

[5] M. Beckerman, M. Salomaa, A. Sperduto, J.D. Molitoris, A. DiRienzo, Phys. Rev. C 25, 837 (1982)

[6] C.H. Dasso, S. Landowne, A. Winther, Nucl. Phys. A 405, 381 (1983)
[7] C.H. Dasso, S. Landowne, A. Winther, Nucl. Phys. A 407, 221 (1983)

[8] R.A. Broglia, C.H. Dasso, S. Landowne, A. Winther, Phys. Rev. C 27, 2433 (1983)

[9] R.A. Broglia, C.H. Dasso, S. Landowne, G. Pollarolo, Phys. Lett. B 133, 34 (1983)

[10] M. Dasgupta, D.J. Hinde, A. Diaz-Torres, B. Bouriquet, C.I. Low, G.J. Milburn, J.O. Newton, Phys. Rev. Lett. 99, 192701 (2007)

[11] T. Ichikawa, K. Matsuyanagi, Phys. Rev. C 92, 021602 (2015)

[12] S. Mişicu, H. Esbensen, Phys. Rev. Lett. 96, 112701 (2006)

[13] C. Simenel, A.S. Umar, K. Godbey, M. Dasgupta, D.J. Hinde, Phys. Rev. C 95, 031601 (2017)

[14] M.D. Rodríguez, M.L. Brown, M. Dasgupta, D.J. Hinde, D.C. Weisser, T. Kibedi, M.A. Lane, P.J. Cherry, A.G. Muirhead, R.B. Turkentine et al., Nucl. Instrum. Meth. A 614, 119 (2010)

[15] I.P. Carter, M.L. Brown, M. Dasgupta, D.J. Hinde, M. Evers, D.H. Luong, A. Wakhle, E. Williams, Determination of the angular distribution of evaporation residues following transmission through the superconducting solenoidal separator SOLITAIRE, in EPJ Web. Conf. (EDP Sciences, 2012), Vol. 35, p. 05003

[16] J.X. Wei, J.R. Leigh, D.C. Weisser, J.O. Newton, S. Elfstrom, J.P. Lestone, J.X. Chen, D.G. Popescu, D.J. Hinde, Nucl. Instrum. Meth. A 306, 557 (1991)

[17] M.L. Brown, Simulation of a gas-filled solenoid: Optimising functionality and expanding applicability (2011)

[18] H.D. Betz, Rev. Mod. Phys. 44, 465 (1972)

[19] V. Weisskopf, Phys. Rev. 52, 295 (1937)

[20] K. Schmidt, Deexcitation mechanisms in compound nucleus reactions (2009)

[21] A. Gavron, Phys. Rev. C 21, 230 (1980)

[22] O.B. Tarasov, D. Bazin, Nucl. Instrum. Meth. B 204, 174 (2003)

[23] H.A. Bethe, J. Ashkin, Experimental nuclear physics 1, 309 (1953)

[24] M. Wang, G. Audi, A.H. Wapstra, F.G. Kondev, M. MacCormick, X. Xu, B. Pfeiffer, Chinese Phys. C 36, 1603 (2012) 